\title{
SPIN-LATTICE RELAXATION IN POTASSIUM CHROMIUM ALUM
}

\author{
J. FLOKSTRA, G. J. GERRITSMA and L. C. van der MAREL \\ Technische Hogeschool Twente, Afdeling der Technische Natuurkunde, Enschede, The Netherlands
}

Received 8 July 1977

Revised 19 September 1977

A comparison is made between the results of low-frequency relaxation measurements on potassium chromium alum placed in liquid helium and in vacuum, respectively. It is found that only a part of the lattice oscillations is involved in the spin-lattice relaxation process. The spin-lattice relaxation time was found after correcting the time constants of the vacuum measurements by experimental shortening factors. The differences between the spin-lattice relaxation time and the time constants from the liquid-helium measurements can be explained for the greater part by the finite thermal conductivity of the liquid helium.

\section{Introduction}

Potassium chromium alum was one of the first compounds studied in magnetic relaxation experiments. The relaxation behaviour of the magnetically, strongly diluted alum was found to be in accordance with established spin-lattice relaxation theories, but up to now the relaxation phenomena in the concentrated salt have not been satisfactorily understood. It is quite possible that thermal conduction in the crystal and the surrounding medium strongly influences the spinlattice relaxation of magnetically concentrated salts, resulting in an observed relaxation time which differs considerably from the spin-lattice relaxation time [1]. Differential susceptibility measurements on samples in direct contact with liquid helium, combined with similar measurements on these samples in vacuum, make it possible to obtain information about the thermal conduction effects as well as about the internal relaxation process [2]. In the present paper we describe the results of such an experiment on undiluted $\mathrm{KCr}$ alum. New effects in spin-lattice relaxation have been found and these will possibly contribute to the understanding of the relaxation phenomena in the concentrated compound.

From crystal structure measurements and electron spin resonance experiments it can be concluded that the $\mathrm{Cr}^{3+}$ ion in $\mathrm{KCr}\left(\mathrm{SO}_{4}\right)_{2} \cdot 12 \mathrm{H}_{2} \mathrm{O}$ is in a cubic elec- trical crystalline field with a small trigonal distortion [3]. By the combined effect of this trigonal field and the spin-orbit coupling the ground quadruplet is split into two doublets with a difference in energy of about $0.15 \mathrm{~cm}^{-1}$. The nearest orbital level is at about $10000 \mathrm{~cm}^{-1}$. Because the zero-field splitting is much less than $k T$ the expression for the direct process relaxation time of this multilevel system is given by [4]

$\tau_{\mathrm{dir}}^{-1}=A H^{3} \operatorname{coth}\left(\mu_{0} g \beta H / 2 k T\right)$,

where $g$ is the spectroscopic splitting factor and $\beta$ the Bohr magneton. The field dependence of eq. (1) was found for cesium chromium alum [5] but up to now not for $\mathrm{KCr}$ alum.

The relaxation behaviour of $\mathrm{KCr}$ alum has been studied by means of various resonant measuring techniques $[6-10]$. The temperature dependence of the direct process was found in the strongly diluted compound. At a higher concentration of magnetic ions it was observed that the temperature dependence of the relaxation time is given by $T^{-\alpha}$, with $\alpha>1$.

Using the low-frequency induction technique Kramers et al. [11] measured a $T^{-3}$ dependence for the relaxation time of a powdered sample of magnetically undiluted $\mathrm{KCr}$ alum at a field of $3370 \mathrm{Oe}$. These non-resonant relaxation measurements were carried further by Van den Broek and Van der Marel [12], 
who affirmed that the direct spin-lattice relaxation process is not predominant in the concentrated salt. They derived the relaxation time constant $\tau_{\text {abs }}$ obtained from the absorption curve in the conventional way proportional to $T^{-3.65}$. Using the Eisenstein model for the relaxation [13] they tried to explain the nonideal shape of the absorption curves which was found for $T>T_{\lambda}$, but in order to obtain a fairly good agreement between calculated and measured curves the value for the thermal conductivity of the lattice had to be taken as considerably smaller than was measured by Bij1 [14]. The ideal shape of the absorption curves for $T<T_{\lambda}$ was thought to be the result of the penetration of superfluid helium into the crystal through narrow channels and cracks, which would greatly improve the heat contact with the cooling liquid.

In a previous paper [1] we examined a thermal conduction model for the relaxation which includes the thermal conduction in the liquid helium. Assuming the direct process for the spin-lattice relaxation and taking the values in ref. 12 for the thermal conductivity of the lattice, it was calculated there that $\tau_{\mathrm{abs}}$ is proportional to $T^{-3.5}$ for $\mathrm{KCr}$ alum. In this paper we present the calculations of the influence of the thermal conduction in the lattice and in the liquid-helium bath based on real experimental values for the thermal transport quantities in the thermal conduction model and the results will be compared with our liquid-helium experiments on $\mathrm{KCr}$ alum (section 4). Intrinsic properties of $\mathrm{KCr}$ alum were derived from the vacuum measurements (sections 2 and 3 ). It will be shown that the rate-determining process in the energy transport is not the thermal conduction in the lattice but that in the liquid-helium bath.

\section{Measurements with the sample in liquid helium and in vacuum, respectively}

Dynamical susceptibility measurements were performed on a cylindrical single crystal of $\mathrm{KCr}$ alum with a radius of $2.3 \mathrm{~mm}$ and a length of about $10 \mathrm{~mm}$ at four different temperatures. The sample was in direct contact with the liquid helium in the first series of measurements and in vacuum (this means in helium gas with a pressure below $10^{-3}$ torr) in the second series. The experimental set-up is described in ref. 15 . The direction of the applied magnetic field with respect to the crystallographic $c$-axis was arbitrary but was the same in both series.

Following the thermodynamic theory of Casimir and Du Pré [16] the dispersion and absorption in the two situations can easily be derived as a function of the frequency. In the case of liquid-helium contact it holds that

$\chi^{\prime} / \chi_{0}=1-F+F /\left(1+\omega^{2} \tau_{\mathrm{s}}^{2}\right)$,

$\chi^{\prime \prime} / \chi_{0}=F \omega \tau_{s} /\left(1+\omega^{2} \tau_{s}^{2}\right)$

and for vacuum conditions

$$
\begin{aligned}
& \chi^{\prime} / \chi_{0}=1-F+\left[C_{\mathrm{L}} /\left(C_{\mathrm{H}}+C_{\mathrm{L}}\right)\right] F /\left(1+\omega^{2} \tau_{\mathrm{v}}^{2}\right), \\
& \chi^{\prime \prime} / \chi_{0}=\left[C_{\mathrm{L}} /\left(C_{\mathrm{H}}+C_{\mathrm{L}}\right)\right] F \omega \tau_{\mathrm{v}} /\left(1+\omega^{2} \tau_{\mathrm{v}}^{2}\right)
\end{aligned}
$$

where $\chi_{0}$ is the isothermal susceptibility at magnetic field $H, F=\left(C_{\mathrm{H}}-C_{\mathrm{M}}\right) / C_{\mathrm{H}}, C_{\mathrm{H}}$ and $C_{\mathrm{M}}$ being the specific heat of the spin system at constant field and magnetization, respectively, $\tau_{\mathrm{s}}$ the spin-lattice relaxation time, and $C_{\mathrm{L}}$ the lattice specific heat. The vacuum relaxation time $\tau_{\mathrm{v}}$ is given by

$\tau_{\mathrm{v}}=\left[C_{\mathrm{L}} /\left(C_{\mathrm{H}}+C_{\mathrm{L}}\right)\right] \tau_{\mathrm{s}}$

where $C_{\mathrm{L}} /\left(C_{\mathrm{H}}+C_{\mathrm{L}}\right)$ is termed the shortening factor of the spin-lattice relaxation time $\tau_{s}$. In the experiments the dispersion and absorption are taken relatively with respect to $\chi_{0}(H=0)$. The low-frequency limit of the dispersion curve of eq. (2a) then equals $\chi_{0}(H)$ / $\chi_{0}(H=0)$ (denoted by $K$, which is smaller than 1 due to paramagnetic saturation effects) and that of eq. (3a) equals $\left[\left(C_{\mathrm{M}}+C_{\mathrm{L}}\right) /\left(C_{\mathrm{II}}+C_{\mathrm{L}}\right)\right] \chi_{0}(H) / \chi_{0}(H=0)$ (denoted by $L$ ). The high-frequency limit is in both cases equal to $\left(C_{\mathrm{M}} / C_{\mathrm{H}}\right) \chi_{0}(H) / \chi_{0}(H=0)$ (denoted by $r$ ).

Usually the experimental dispersion and absorption curves do not have the ideal shape as described by eqs. (2) and (3). The deviation from the ideal curves is expressed by the deviation parameter which is given by $d_{1 \mathrm{iq}}=1-2 h /(K-r)$ for the liquid-helium susceptibility curves and by $d_{\mathrm{vac}}=1-2 h /(L-r)$ for the vacuum curves where $h$ is the maximum value of the corresponding absorption curve. The relaxation time constant is taken from the frequency at which the absorption curve has its maximum. For the liquid. helium measurements this time constant is denoted by $\tau_{\text {liq }}$ and for the vacuum measurements by $\tau_{\text {vac }}$. 
These two experimental time constants are not necessarily equal to $\tau_{\mathrm{s}}$ and $\tau_{\mathrm{v}}$ in the Casimir and Du Pré theory, respectively. The results for $\tau_{\text {liq }}, \tau_{\text {vac }}, d_{1 \text { liq }}$ and $d_{\text {vac }}$ are given in figs. $1-4$. It is seen that the deviation parameters in the weak-field range are equal in both series of measurements. In the strong-field range $(H>5 \mathrm{kOe})$, however, deviations are present in the

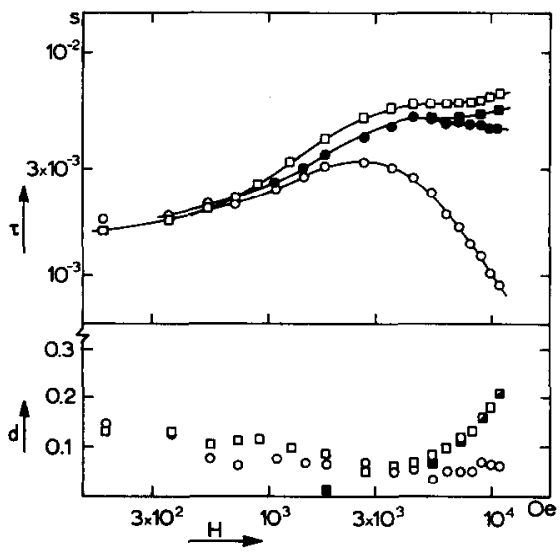

Fig. 1. The field dependence of $\tau$ and $d$ of $\mathrm{KCr}$ alum at $4.21 \mathrm{~K}$ : liquid-helium contact, $\square, \tau_{\text {liq }}, d_{\text {liq }}$; vacuum, $O, \tau_{\text {vac }}, d_{\text {vac }}$; $\tau_{\text {vac }}$ divided by $C_{\mathrm{L}} /\left(C_{\mathrm{H}}+C_{\mathrm{L}}\right), \bullet, \tau_{\mathrm{s} 1}$; calculated with $\tau_{\mathrm{s} 1}$, $\boldsymbol{n}, \tau_{\mathrm{abs}}, d_{\mathrm{abs}}$.

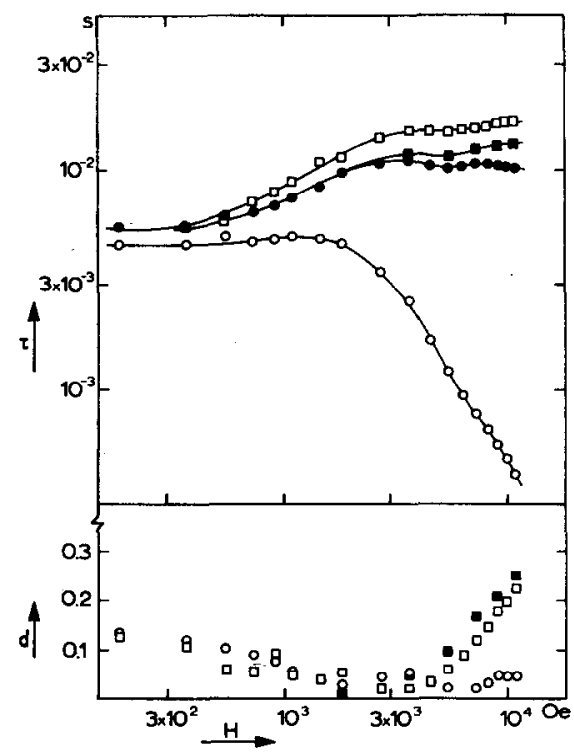

Fig. 2. The field dependence of $\tau$ and $d$ of $\mathrm{KCr}$ alum at $3.03 \mathrm{~K}$. Symbols according to those in fig. 1 .

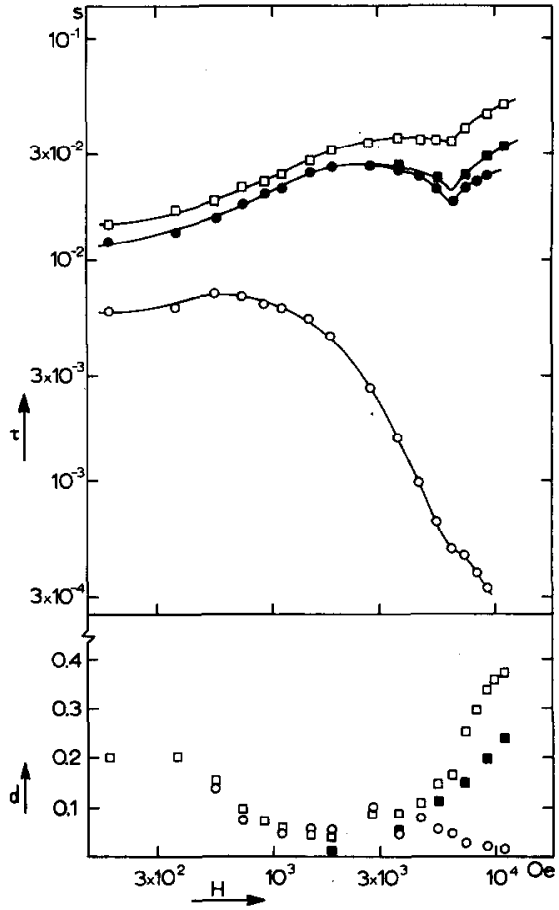

Fig. 3. The field dependence of $\tau$ and $d$ of $\mathrm{KCr}$ alum at $2.27 \mathrm{~K}$. Symbols according to those in fig. 1 .

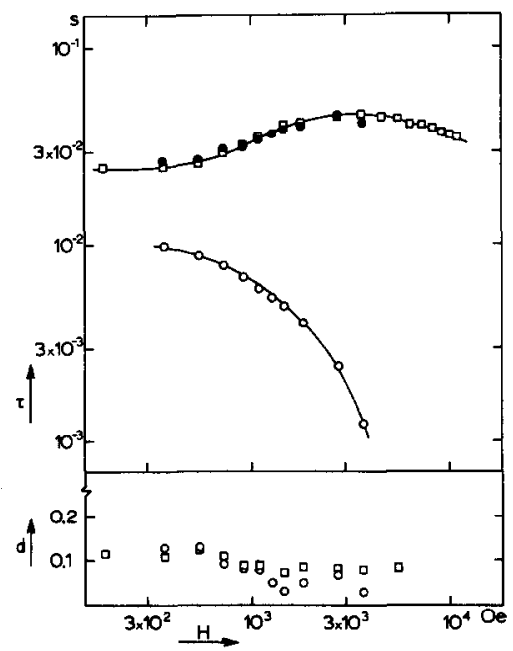

Fig. 4. The field dependence of $\tau$ and $d$ of $\mathrm{KCr}$ alum at $2.00 \mathrm{~K}$. Symbols according to those in fig. 1 . There is no difference between $\tau_{\mathrm{abs}}$ and $\tau_{\mathrm{s} 1}$. 
liquid-helium susceptibility curves but they are absent in the vacuum curves. The time constants $\tau_{\mathrm{liq}}$ and $\tau_{\mathrm{vac}}$ may differ greatly but in order to compare the vacuum results with those of the liquid-helium measurements,

$\tau_{\text {vac }}$ has to be divided by the shortening factor (see section 3 ). At about $6.5 \mathrm{kOe}$ both the $\tau_{\text {liq }}$ vs. $H$ and the $\tau_{\text {vac }}$ vs. $H$ curves show an anomaly, possibly due to cross-relaxation effects [4].

From the high-frequency limit of the dispersion curve [equal to $C_{\mathrm{M}} / C_{\mathrm{H}}$ with $C_{\mathrm{M}}=b / T^{2}$ and $C_{\mathrm{II}}=\left(b+\mu_{0} C H^{2}\right) / T^{2}, C$ is the Curie constant $]$ the $b / \mu_{0} C$ value could be derived as $4.3 \times 10^{9} \mathrm{~A}^{2} / \mathrm{m}^{2}$ $\left(=6.8 \times 10^{5} \mathrm{Oe}^{2}\right)$ which is in good agreement with the results of Van den Broek et al. [12]. Using the value $\mu_{0} C=109 \times 10^{-9} \mathrm{JK} / \mathrm{A}^{2} \mathrm{~m}$, the specific heat $C_{\mathrm{M}}$ is obtained as $468 / \mathrm{T}^{2}\left(\mathrm{~J} / \mathrm{m}^{3} \mathrm{~K}\right)$.

The maximum of the vacuum absorption curve becomes very small at strong fields and low temperatures. For example, $\chi_{\max }^{\prime \prime} / \chi_{0} \approx 0.01$ at $H=3.6 \mathrm{kOe}$ and $T=2.00 \mathrm{~K}$. This is the reason that the field range for which $\tau_{\text {vac }}$ could be determined is rather restricted.

\section{The shortening factors}

The spin-lattice relaxation time constant can be obtained by dividing the experimental vacuum time constant $\tau_{\text {vac }}$ by the shortening factor $C_{\mathrm{L}} /\left(C_{\mathrm{H}}+C_{\mathrm{L}}\right)$. The first mentioned time constant is denoted by $\tau_{\text {sl }}$ because this quantity is not necessarily equal to $\tau_{s}$ ( $\tau_{\mathrm{s}}$ and $\tau_{\mathrm{v}}$ are related to the Casimir and Du Pré theory). $C_{\mathrm{H}}$ is calculated with the data from the preceding section and $C_{\mathrm{L}}$ can be taken from the literature as $C_{\mathrm{L}}=15.07 T^{3}\left(\mathrm{~J} / \mathrm{m}^{3} \mathrm{~K}\right)[17]$. Using these values it is found that $\tau_{\mathrm{sl}}$ differs greatly from $\tau_{\text {liq }}$, especially at $T=3.03 \mathrm{~K}, 2.27 \mathrm{~K}$ and $2.00 \mathrm{~K}$ and even at weak magnetic fields. The low-frequency limit $L$ of the vacuum dispersion curve calculated from $K\left(C_{\mathrm{M}}+C_{\mathrm{L}}\right)$ / $\left(C_{\mathrm{H}}+C_{\mathrm{L}}\right)$ is considerably larger than the value obtained from the vacuum measurements. Therefore another method is followed for the derivation of the shortening factors.

Using the definitions for $K, L$ and $r$ (section 2) it can be shown that

$C_{\mathrm{M}} / C_{\mathrm{L}}=(r / K)(K-L) /(L-r)$,

$C_{\mathrm{H}} / C_{\mathrm{L}}=(K-L) /(L-r)$.
The ratios of the specific heats are now expressed in experimentally observable quantities. The shortening factors can be derived using eq. ( $5 b$ ), hence the vacuum time constants are now corrected by experimentally obtained values for $C_{\mathrm{L}} /\left(C_{\mathrm{H}}+C_{\mathrm{L}}\right)$.

It is found that at $4.21 \mathrm{~K}$ the value of $C_{\mathrm{M}} / C_{\mathrm{L}}$ is slightly dependent on the field, contrary to theoretical predictions. Because $r$ agrees very well with $K C_{\mathrm{M}} / C_{\mathrm{H}}$, which is calculated with $b=468 \mathrm{JK} / \mathrm{m}^{3}, \mu_{0} C=109$ $\times 10^{-9} \mathrm{JK} / \mathrm{A}^{2} \mathrm{~m}$ and the expression for $K$ in the molecular field theory [18], $C_{\mathrm{L}}$ seems to be more or less dependent on the field. The results for $C_{\mathrm{L}}$ are presented in fig. 5 . It is worth mentioning that the maximum value of $C_{\mathrm{L}}$ in fig. $5\left(1120 \mathrm{~J} / \mathrm{m}^{3} \mathrm{~K}\right)$ is equal to the value obtained by Kapadnis [17] by means of heat-capacity measurements. The average value of $C_{\mathrm{L}}$ in the figure is about $1000 \mathrm{~J} / \mathrm{m}^{3} \mathrm{~K}$, so with respect to Kapadnis's value, about $90 \%$ of the lattice specific heat is involved in the spin-lattice relaxation at $4.2 \mathrm{~K}$. At the other temperatures $C_{\mathrm{L}}$ also seems to be more or less dependent on the field in a manner similar to that

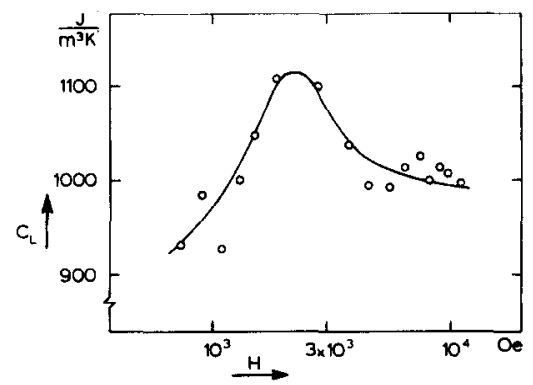

Fig. 5. The specific heat of the part of the lattice which is involved in the spin-lattice relaxation as a function of the magnetic field at $T=4.21 \mathrm{~K}$. The specific-heat value measured by Kapadnis is $1120 \mathrm{~J} / \mathrm{m}^{3} \mathrm{~K}$.

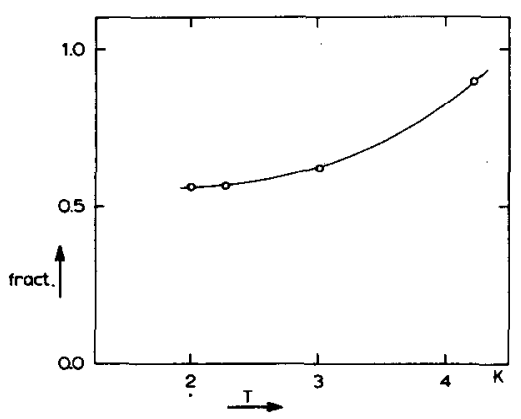

Fig. 6. The average fraction of the lattice specific heat involved in the spin-lattice relaxation as a function of the temperature. 
in fig. 5 , but the change in $C_{\mathrm{L}}$ is not more than $10 \%$. In fig. 6 the average fraction of the lattice specific heat involved in the spin-lattice relaxation is presented as a function of the temperature. At $T=2 \mathrm{~K}$ this fraction amounts to about 0.6 . Obviously, when the temperature decreases a smaller part of the lattice oscillations is involved in the spin-lattice relaxation.

Using the experimental shortening factors $\tau_{\mathrm{sl}}$ was calculated and the results are presented in figs. 1-4. It is seen that $\tau_{\mathrm{sl}}$ lies somewhat below $\tau_{\text {liq }}$ over a large field range and that the difference between $\tau_{\text {sl }}$ and $\tau_{\text {liq }}$ increases with increasing field. At $T=2.00 \mathrm{~K}$, $\tau_{\text {sl }}$ coincides with $\tau_{\text {liq }}$ over the whole field range for $\tau_{\text {vac }}$. This last result indicates that the thermal conductivity of the lattice does not obscure the spinlattice relaxation. It can be expected that the liquidhelium bath plays a part in the relaxation process for $T>T_{\lambda}$ resulting in a difference between $\tau_{\mathrm{sl}}$ and $\tau_{\text {liq }}$. In the next section the role of the environment will be analysed.

\section{Calculations based on the thermal conduction model}

The influence of the heat conduction in the crystal and the bath on the time constant $\tau_{\text {abs }}$ obtained from the absorption curve was calculated with the thermal conduction model dealt with in ref. 1 . It was assumed that the shape of the sample could be approximated by a sphere with a radius of $2.3 \mathrm{~mm}$. The values for the specific heats $C_{\mathrm{M}}, C_{\mathrm{H}}$ and $C_{\mathrm{L}}$ (the effective value) of $\mathrm{KCr}$ alum were derived from our experiments. The heat transfer coefficient $\alpha\left(=C_{\mathrm{H}} / \tau_{\mathrm{sl}}\right)$ was determined using the values of $\tau_{\mathrm{sl}}$ in figs. $1-4$. The thermal conductivity of the lattice was taken from Bijl's measurements [14], who found that $\lambda_{\mathrm{L}}$ was proportional to $T^{2}$ in the liquid-helium temperature range. The magnitude of $\lambda_{L}$ is dependent on the manner in which the sample is cooled and therefore the average value of $30 \mathrm{~W} / \mathrm{Km}$ was taken for it at $4.2 \mathrm{~K}$. In a forthcoming paper [15] it will be demonstrated that the thermal conduction in the lattice is not the rate-determining factor for the relaxation behaviour so it is not necessary to consider which part of the total thermal conductivity should be taken if the phonon system does not participate in the heat transport as a whole. The heat properties of liquid helium are given in the literature [19]. The thermal resistance $R_{K}$ between the crystal and the liquid-helium bath was taken as zero.

The time constants $\tau_{\text {abs }}$ and the deviation parameters $d$ derived from the calculated absorption curves are presented in figs. $1-3$ for $T=4.21 \mathrm{~K}, 3.03 \mathrm{~K}$ and $2.27 \mathrm{~K}$, respectively. It can be seen that $\tau_{\text {abs }}>\tau_{\text {sl }}$ for $H>4 \mathrm{kOe}$ and that the difference between these two time constants increases with increasing field.

Obviously, the thermal properties of the liquid-helium bath influence the relaxation behaviour for $H>4 \mathrm{kOe}$. The calculated deviation parameters at $4.21 \mathrm{~K}$ and $3.03 \mathrm{~K}$ agree well with the experimental ones in the strong-field range but at $T=2.27 \mathrm{~K}$ the agreement is rather poor. At weak magnetic fields the calculated $d$ values are zero. At $2.00 \mathrm{~K}$ no difference is calculated between $\tau_{\mathrm{sl}}$ and $\tau_{\mathrm{abs}}$, which is the result of the large thermal conductivity of the lattice and the bath. At this temperature $\tau_{\text {abs }}$ coincides with the experimental time constants $\tau_{\text {liq }}$, but for $T>T_{\lambda}$ there still remains a difference between $\tau_{\text {abs }}$ and $\tau_{\text {liq }}$. This difference could not be attributed to a thermal resistance between crystal and bath.

\section{The spin-lattice relaxation time}

The field dependence of the direct spin--lattice relaxation process according to eq. (1) is not found in the field range up to $10 \mathrm{kOe}$. In the weak-field range physical imperfections and magnetic impurities may cause a rapid energy exchange between the spin system and the lattice leading to a field dependence of the Brons-Van Vleck type [20].

The temperature dependence of $\tau_{\mathrm{sl}}$ and of $\tau_{\text {liq }}$ is presented for two fields in fig. 7. It is seen that for both fields the four $\tau_{1 \text { iq }}$ values do not lie on a straight line so that it may be expected that the $\tau_{\text {liq }}$ vs. $T$ curve has a discontinuity at $T=T_{\lambda}$. However, the four $\tau_{\mathrm{sl}}$ values (at $T=2.00 \mathrm{~K}, \tau_{\mathrm{sl}}=\tau_{\text {liq }}$ ) do lie on a straight line, which demonstrates that the correction method applied to the vacuum time constants has been carried out correctly. The spin-lattice relaxation time $\tau_{\mathrm{sl}}$ is proportional to $T^{-3.3}$ at $H=1.8 \mathrm{kOe}$ and to $T^{-2.7}$ at $11 \mathrm{kOe}$. For $T>T_{\lambda}$ it is found that $\tau_{\text {liq }} \propto T^{-3.3}$ at 1.8 and $11 \mathrm{kOe}$. Hence, the temperature dependence of $\tau_{1 \mathrm{iq}}$ is almost equal to that of $\tau_{\mathrm{sl}}$. This implies that the temperature dependence of the relaxation time measured by Kramers et al. [11] and Van den Broek et al. [12] is for the greater part due 


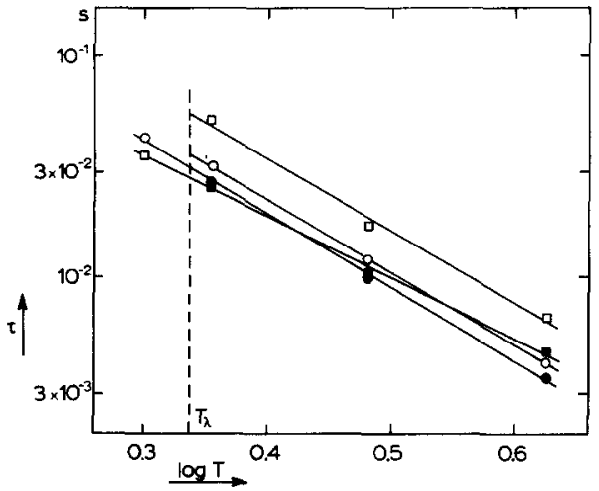

Fig. 7. $\tau$ vs. $T$ curves of $\mathrm{KCr}$ alum; $H=1.8 \mathrm{kOe}: 0, \tau_{\mathrm{liq}} ; \bullet, \tau_{\mathrm{sl}}$; $H=11 \mathrm{kOe:} \square, \tau_{\mathrm{liq}} ; \boldsymbol{\square}, \tau_{\mathrm{sl}}$. The discontinuity in the $\tau_{\text {liq }}$ vs. $T$ curve at $T=T_{\lambda}$ is not present in the $\tau_{\mathrm{sl}}$ vs. $T$ curve.

to an internal relaxation process. The magnitude of their time constants, however, is influenced by thermal conduction effects in the liquid-helium bath. Obviously, the direct process is not predominant in $\mathrm{KCr}$ alum in the temperature range from 2 to $4.2 \mathrm{~K}$ for $H<10 \mathrm{kOe}$. The fact that not all the lattice oscillations are involved in the spin-lattice relaxation may be an indication that relaxation mechanisms in the phonon system influence the observed relaxation time constant.

\section{Conclusions}

From the low-frequency final values of the vacuum dispersion curves it is found that the entire lattice of $\mathrm{KCr}$ alum is not effective in the relaxation. At $4.2 \mathrm{~K}$ about $90 \%$ and at $2 \mathrm{~K}$ about $60 \%$ of the lattice specific heat is involved in the spin-lattice relaxation. This effect seems to be slightly dependent on the field. Equilibrium within the whole lattice system was not observed so that it can be concluded from the lowfrequency limit of the apparatus $(1.5 \mathrm{~Hz})$ that the time constant of this lattice relaxation process is larger than $100 \mathrm{~ms}$. It is evident that the partial involvement of the lattice cannot be investigated in relaxation measurements with the sample in liquid helium. Recent measurements which we performed on cerium magnesium nitrate showed that the lattice of this compound is also partially involved in the relaxation process while the temperature dependence of the lattice fraction is similar to that in fig. 6.

From the comparison of the deviation parameters of the susceptibility measurements with the sample in liquid helium and in vacuum, respectively, it can be concluded that the deviations in the weak-field range are the result of internal processes and that the large deviations of the liquid-helium susceptibility curves in the strong-field range are due to thermal conduction effects. From calculations based on the thermal conduction model it can be concluded that these strongfield deviations are due to the bad thermal conduction in the liquid helium and that the thermal conduction in the lattice plays no part.

From vacuum measurements the spin-lattice relaxation time $\tau_{\mathrm{s} 1}$ can be derived if the time constants $\tau_{\text {vac }}$ are divided by the experimental $C_{\mathrm{L}} /\left(C_{\mathrm{H}}+C_{\mathrm{L}}\right)$ values. In contrast to the $\tau_{\text {liq }}$ vs. $T$ curve, the $\tau_{\mathrm{sl}}$ vs. $T$ curve does not show a discontinuity at $T=T_{\lambda}$, thus confirming that the calculation of $\tau_{\mathrm{s}}$ from $\tau_{\mathrm{vac}}$ has been carried out correctly. The differences between $\tau_{\text {sl }}$ and $\tau_{\text {liq }}$ could be partially explained with the thermal conduction model. Just as with the liquid-helium measurements the vacuum relaxation measuring technique also leads to a strong temperature dependence for the spin-lattice relaxation time، So it is not the thermal conduction that causes this strong temperature dependent behaviour. The present vacuum measurements show that it is worthwhile carefully examining the role of the lattice in the relaxation process.

\section{References}

[1] J. Flokstra, G. J. Gerritsma, G. A. Hartemink and L. C. van der Marel, Physica 77 (1974) 99.

[2] J. Flokstra, G. J. Gerritsma, C. L. M. Pouw and A. J. van Duyneveldt, Physica 92B (1977) 339.

[3] C. A. Beevers and H. Lipson, Proc. Roy. Soc. A146 (1934) 570 .

B. Bleaney, Proc. Roy. Soc. A204 (1950) 203.

[4] See, for instance, J. C. Verstelle and D. A. Curtis, Handbuch der Physik, vol. 18 (1968) p. 1.

[5] H. M. C. Eijkeihof, C. L. M. Pouw and A. J. van Duyneveldt, Physica 62 (1972) 257.

[6] A. H. Eschenfelder and R. T. Weidner, Phys. Rev. 92 (1953) 869 .

[7] B. Bölger, J. M. Noothoven van Goor and K. J. Damma, Physica 27 (1961) 18.

[8] Y. Nisida, J. Phys. Soc. Jap. 19 (1964) 2273.

[9] K. J. Standley and-A. O. Tooke, J. Phys. C1 (1968) 149.

[10] G. A. Candela, J. Chem. Phys. 52 (1970) 3754.

[11] H. C. Kramers, D. Bijl and C. J. Gorter, Physica 16 (1950) 65 . 
[12] J. van den Broek, L. C. van der Marel and C. J. Gorter, Physica 27 (1961) 661.

[13] J. Eisenstein, Phys. Rev. 84 (1951) 548.

[14] D. Bijl, Physica 14 (1949) 684.

[15] G. J. Gerritsma, J. Flokstra, A. J. W. A. Vermeulen, J. J. M. Scholten and L. C. van der Marel, to be published.

[16] H. B. G. Casimir and F. K. Du Pré, Physica 5 (1938) 507. H. B. G. Casimir, Physica 6 (1939) 156.
[17] D. G. Kapadnis, Physica 22 (1956) 159.

[18] J. Flokstra, H. C. Meijer, G. J. C. Bots, W. A. Verheij and L. C. van der Marel, Physica 63 (1973) 288.

[19] See, for instance, J. Wilks, The Properties of Liquid and Solid Helium (Clarendon Press, Oxford, 1967).

[20] J. H. van Vleck, Phys. Rev. 57 (1940) 426.

C. L. M. Pouw, Thesis, Leiden (1975). 